\title{
Ferro I phase and gadolinium
}

\author{
J. F. Collingwood* \\ Institute of Science and Technology in Medicine, Keele University, Stoke-on-Trent, Staffordshire ST4 7QB, United Kingdom \\ S. B. Palmer and M. R. Lees \\ Department of Physics, University of Warwick, Coventry CV4 7AL, United Kingdom \\ C. Edwards \\ NDT Consultants Ltd., Coventry CV3 4FJ, United Kingdom
}

J. A. Santos and J. B. Sousa

IFIMUP, Universidade do Porto, 4169-007 Porto, Portugal

D. C. Jiles

Wolfson Centre for Magnetics, Cardiff University, Cardiff CF24 3AA, United Kingdom

(Received 25 September 2008; revised manuscript received 21 January 2009; published 3 March 2009)

\begin{abstract}
Certain Gd alloys, such as Gd-Y, are known to exhibit an apparent "double ferromagnetism" in which the $c$ axis component orders on cooling from the paramagnetic phase $\left(T_{C 1}\right)$, while the basal-plane component remains effectively paramagnetic. Subsequently the basal-plane component orders at a lower temperature $\left(T_{C 2}\right)$. Our measurements of low-field ac susceptibility and magnetization for high-quality Gd and Gd-Y single crystals suggest that either the behavior originally used to identify ferro I, namely, the concave-upward growth of the $a$ axis moment in the approach on cooling to $T_{\mathrm{SR}}$, is not necessarily an indicator of the phase, or that ferro $\mathrm{I}$ is also present in pure Gd. Of the several models that exist to describe ferro I, the results from this study favor the random-cone model.
\end{abstract}

DOI: 10.1103/PhysRevB.79.104401

PACS number(s): $75.50 . \mathrm{Cc}, 75.30 . \mathrm{Cr}, 75.30 . \mathrm{Kz}$

\section{INTRODUCTION}

Gadolinium and the gadolinium-yttrium alloy system exhibit an interesting range of magnetic ordering behavior, including ferromagnetic structures and helical antiferromagnetism. Perhaps the least understood of the magnetic phases in Gd-Y is that termed ferro I. The ferro I phase was first identified by Legvold and co-workers ${ }^{1,2}$ on the basis of the magnetic ordering behavior of Gd-Y single crystals. Typical Curie-Weiss-type concave-upward growth, an upward divergence from a simple linear relationship in the transition from the paramagnetic to ferromagnetic phase, is seen in the $c$ axis magnetization at $T_{C 1}$, and the same upward growth then occurs at a significantly lower temperature $\left(T_{C 2}\right)$ for the $a$ axis crystal as the system orders into the simple canted (ferro II) phase.

Pure Gd orders initially as a $c$ axis ferromagnet with a Curie temperature $\left(T_{C}\right) \sim 293 \mathrm{~K}$ and a spin-reorientation transition at $T_{\mathrm{SR}} \sim 225 \mathrm{~K}$, where the moments cant away from the $c$ axis at an angle $\theta$, and $\theta(T)$ varies smoothly below $T_{\mathrm{SR}}$. It is currently accepted that in Gd-Y, ferro I develops for alloys with $\mathrm{Y} \geq 10 \%$, and heat-capacity data suggest that the transition at $T_{C 2}$ is first order in Gd-Y crystals with $Y=26$ and 30 at. \%. It was suggested that this decoupling of the ferro I phase from ferro II would break down in the approach to pure $\mathrm{Gd},{ }^{3}$ where $\mathrm{Gd}-\mathrm{Y}$ with $\mathrm{Y} \leq 10 \%$ has been assumed to order like pure $\mathrm{Gd}$ in the higher temperature ordered phase. $^{2}$

On the assumption that the role of $\mathrm{Y}$ in ferro I is largely that of a nonmagnetic diluent, it was expected that Gd-Sc, Gd-Lu, and Gd-La might exhibit similar behavior. Subse- quent single-crystal studies of the magnetization revealed ferro $\mathrm{I}$ in $\mathrm{Gd}_{80} \mathrm{Lu}_{20}$ over a narrow temperature interval and in $\mathrm{Gd}_{75} \mathrm{Y}_{17.5} \mathrm{Lu}_{7.5}$ over a significantly broader interval. ${ }^{4}$ Studies of $\mathrm{Gd}-\mathrm{Sc}$ have revealed no direct evidence for the presence of ferro I. ${ }^{5}$

In ferro I, the basal-plane component is expected to exhibit behavior consistent with an order-disorder ferromagnetic transition at $T_{C 2}$, since the main feature of ferro $\mathrm{I}$ is the paramagneticlike response of the basal-plane moment in a region where the $c$ axis component is ordered. When ferro I was originally proposed, the main features used to differentiate between the simple ferromagnetic behavior of $\mathrm{Gd}$ and ferro I in Gd-Y were the concave-downward growth (a downward divergence from a linear relationship on moving from the higher- to the lower-temperature ordered phase) of the $a$ axis magnetization in $\mathrm{Gd}$ on the approach to $T_{C 2}$ and the concave-upward growth in Gd-Y. ${ }^{1,2}$ It is accepted that the behavior in $\mathrm{Gd}$ originates with the higher-order anisotropy terms that develop on cooling, forcing the easy direction of magnetization away from the $c$ axis. Meanwhile, the concave-upward growth seen in the alloys follows the CurieWeiss form for a disorder-order transition, a strong indication that the basal-plane component behaves paramagnetically until it orders at $T_{C 2}$. The nature of ferro I was subsequently explored in neutron-diffraction studies of Gd-Y alloys, and the absence of order in the basal plane until in the close approach to $T_{C 2}$ was confirmed directly for samples with $\mathrm{Y}$ $>29 \%$. $^{6}$

Legvold et al. ${ }^{1}$ proposed the random-cone virtual-crystal model to account for the double ferromagnetism in the ferro I phase. Here, the moments lie on the surface of a cone about 
TABLE I. The shape demagnetizing factor, $N$, determined using the length/diameter of each needle crystal for a range of susceptibilities $(\chi)$, where $N$ corresponds to $N_{m}$ as calculated by Chen et al. (Ref. 9) for the principal axis of each crystal.

\begin{tabular}{lccccc}
\hline \hline & & \multicolumn{3}{c}{$\mathrm{N}$} \\
\cline { 3 - 5 } Sample & Needle axis & $\chi=0$ & $\chi=1$ & $\chi=10$ & $\chi=\infty$ \\
\hline $\mathrm{Gd}$ & $c$ & 0.062 & 0.051 & 0.039 & 0.033 \\
$\mathrm{Gd}$ & $a$ & 0.068 & 0.056 & 0.043 & 0.037 \\
$\mathrm{Gd}_{99.0} \mathrm{Y}_{1.0}$ & $c$ & 0.056 & 0.046 & 0.034 & 0.028 \\
$\mathrm{Gd}_{99.0} \mathrm{Y}_{1.0}$ & $a$ & 0.064 & 0.053 & 0.040 & 0.034 \\
$\mathrm{Gd}_{97.5} \mathrm{Y}_{2.5}$ & $c$ & 0.075 & 0.062 & 0.048 & 0.042 \\
$\mathrm{Gd}_{97.5} \mathrm{Y}_{2.5}$ & $a$ & 0.048 & 0.039 & 0.029 & 0.023 \\
$\mathrm{Gd}_{95.0} \mathrm{Y}_{5.0}$ & $c$ & 0.048 & 0.039 & 0.029 & 0.023 \\
$\mathrm{Gd}_{95.0} \mathrm{Y}_{5.0}$ & $a$ & 0.053 & 0.043 & 0.032 & 0.026 \\
$\mathrm{Gd}_{87.5} \mathrm{Y}_{12.5}$ & $c$ & 0.053 & 0.043 & 0.032 & 0.026 \\
$\mathrm{Gd}_{87.5} \mathrm{Y}_{12.5}$ & $a$ & 0.052 & 0.042 & 0.031 & 0.025 \\
$\operatorname{Gd}_{87.5} \mathrm{Y}_{12.5}$ & $b$ & 0.054 & 0.044 & 0.033 & 0.027 \\
\hline \hline
\end{tabular}

the $c$ axis but are free to move on the surface of the cone so that the component of the moment in the basal plane is disordered. The cone angle for each ion is determined by the average angle of the system as a whole. It has also been proposed that chemical concentration variations, both on the macroscopic and microscopic scales, may be responsible for the double ferromagnetism. ${ }^{6}$ On the macroscopic scale it was suggested that regions within a single Gd-Y crystal that are predominantly Gd could order into a $c$ axis ferromagnet at $T_{C 1}$ and Y-richer regions within the crystal would remain paramagnetic. This simple model has largely been dismissed as such behavior would lead to significant broadening of transitions, as well as sample-to-sample variations and temperature regions where phases coexist, and such behavior is inconsistent with observations. Variations on the microscopic scale have also been considered. It was argued, based on the suggestion that the mean-free path of the conduction electrons would shorten with alloying, that competing Ruderman-Kittel-Kasuya-Yoshida (RKKY) magnetic exchange interactions and frustration could give rise to the behavior seen in the ferro I phase. ${ }^{6}$ The statistical distribution of the ions plays a role in this model, where site-to-site variations in the local anisotropy and exchange energy would lead to a higher ordering temperature for Gd-dominated Gd ions, which favor $c$ axis ferromagnetism, and a lower ordering temperature for the Y-dominated ions where the exchange is weaker, and favors basal-plane antiferromagnetism. The most recent study of ferro I in Gd-Y was carried out using $\mu$ SR (Ref. 7); however these initial data did not enable strong discrimination between the random-cone and chemical concentration models. In this paper the ac susceptibility and dc magnetization properties of the initial ordered region in Gd and Gd-Y are presented, using high-quality singlecrystal solid-solution alloy samples with low demagnetizing factors, in order to determine how the magnetic ordering in the Gd-rich Gd-Y alloys differs to that in pure Gd.

\section{EXPERIMENT}

\section{A. Sample growth and purity}

Long $c$ axis and $a$ axis aligned needle crystals of $\mathrm{Gd}$, $\operatorname{Gd}_{99.0} \mathrm{Y}_{1.0}, \mathrm{Gd}_{97.5} \mathrm{Y}_{2.5}, \mathrm{Gd}_{95.0} \mathrm{Y}_{5.0}$, and $\mathrm{Gd}_{87.5} \mathrm{Y}_{12.5}$, with shape demagnetizing factors $(N)$ as shown in Table I, were used to obtain the data presented in this paper. The single crystals were grown by a solid-state strain-anneal-type technique whereby ingots that had been quickly cooled from the melt, after having been remelted several times to ensure homogeneity, were annealed for approximately $50 \mathrm{~h}$ at a temperature slightly below the hcp to bcc transformation. The needles were then spark-cut from Laue-orientated large single-crystal domains. All the measurements presented in this paper were performed within two years of the sample preparation. Further details of the techniques used to prepare the single crystals are given elsewhere. ${ }^{8}$ The start materials were provided by the Ames Laboratory, Iowa State University, with $99.90 \%$ pure Gd and $99.99 \%$ pure Y. The principal contaminants for the start materials were oxygen $(670 \mathrm{ppm}$ in $\mathrm{Gd} ; 34 \mathrm{ppm}$ in $\mathrm{Y}$ ) and carbon (210 ppm in Gd; $53 \mathrm{ppm}$ in $\mathrm{Y})$.

\section{B. ac susceptibility}

The ac susceptibility measurements were performed using a susceptometer with a standard design that has a primary induction coil and two secondary detection coils cowound with the primary. The configuration allowed the sample to be moved along the axis of the coils from one detection coil to the other while remaining in the longer induction coil. The phase angle between the real and imaginary components, $\chi^{\prime}$ and $\chi^{\prime \prime}$, was adjusted in the paramagnetic region to maximize $\chi^{\prime}$ and minimize $\chi^{\prime \prime}$. The ac field was applied along the long axis of each needle sample. Each needle was bonded to the rigid Tufnol sample-holder rod with GE varnish, with the long axis of the crystal aligned with the holder to within $2^{\circ}$. 
This susceptibility study enabled the magnetic behavior of the samples to be investigated in the absence of an applied dc field. A small ac field of $H_{\omega}=40 \mathrm{~A} \mathrm{~m}^{-1}$, with frequency of $113 \mathrm{~Hz}$, was used to examine the low-field response for each composition over the temperature range of 4.2-305 $\mathrm{K}$. The susceptibility data were converted to SI units on the basis of a calibration performed using $\mathrm{Gd}_{2} \mathrm{O}_{3}$.

\section{Superconducting quantum interference device magnetometry}

The low-field dc magnetization measurements were performed using a Quantum Design MPMS5 superconducting quantum interference device (SQUID) magnetometer, with the field applied parallel to the principal needle axis. For measurements on the $\mathrm{Gd} a$ axis crystal, the sample was warmed to $310 \mathrm{~K}$ and then field-cooled, with the data collected on cooling. For measurements of the $\mathrm{Gd}_{97.5} \mathrm{Y}_{2.5} a$ axis crystal, the sample was zero-field cooled and the data were collected on warming.

\section{RESULTS}

\section{A. ac susceptibility}

The external ac susceptibility is shown for $a, b$, and $c$ axis aligned needles of $\operatorname{Gd}_{87.5} \mathrm{Y}_{12.5}$ in Figs. 1 and 2. According to Ito et al. ${ }^{2}$ the first ordered phase in this alloy should be ferro I, and the susceptibility data are consistent with this. The slight growth in the basal-plane susceptibility at $T_{C 1}$ is followed by the ferro I signature; concave-upward growth approaches the demagnetizing limit $(1 / N)$ at the lower ordering temperature $T_{C 2}$. The sharp spike seen in $\chi_{c}^{\prime}$ at $T_{C 1}$ is attributed to the Hopkinson effect ${ }^{10}$ and is suppressed accordingly by increasing the applied field. The lower-temperature feature at $T^{*}$, which is particularly evident in the $c$ axis data, is discussed elsewhere. ${ }^{11,12}$

In Fig. 3 we present the susceptibility for the $\operatorname{Gd}_{95.0} \mathrm{Y}_{5.0}$ alloy. Here, although the changes in the $c$ axis susceptibility are less pronounced below $T_{C 2}$, the trends appear consistent with those in the $\operatorname{Gd}_{87.5} \mathrm{Y}_{12.5}$, and there is nothing to suggest that the nature of the magnetically ordered regions differs significantly between the compositions.

If we then examine data from $c$ and $a$ axis aligned samples of Gd in Fig. 4, it becomes evident that the concaveupward growth is a feature of the basal plane in the pure element as well, and while the $c$ axis behavior below $T_{C 2}$ differs from that seen in the Gd-Y, the behavior above $T_{C 2}$ shows the same qualitative characteristics. [In former published work (Ref. 11), the ac susceptibility data from the $a$ axis $\operatorname{Gd}_{92.5} \mathrm{Y}_{7.5}$ contained a depression between $T_{C 2}$ and $T_{C 1}$ which was confirmed to be due to a small $c$ axis aligned grain included in the end of the needle.] It should be noted that here we extend the use of the $T_{C 1}$ and $T_{C 2}$ notations to $\mathrm{Gd}$ for the order-disorder and order-order transitions, respectively.

The slight hysteresis evident on warming and cooling is attributed to instrumentation. The temperature sensor was not in direct contact with the sample, and slight variations in
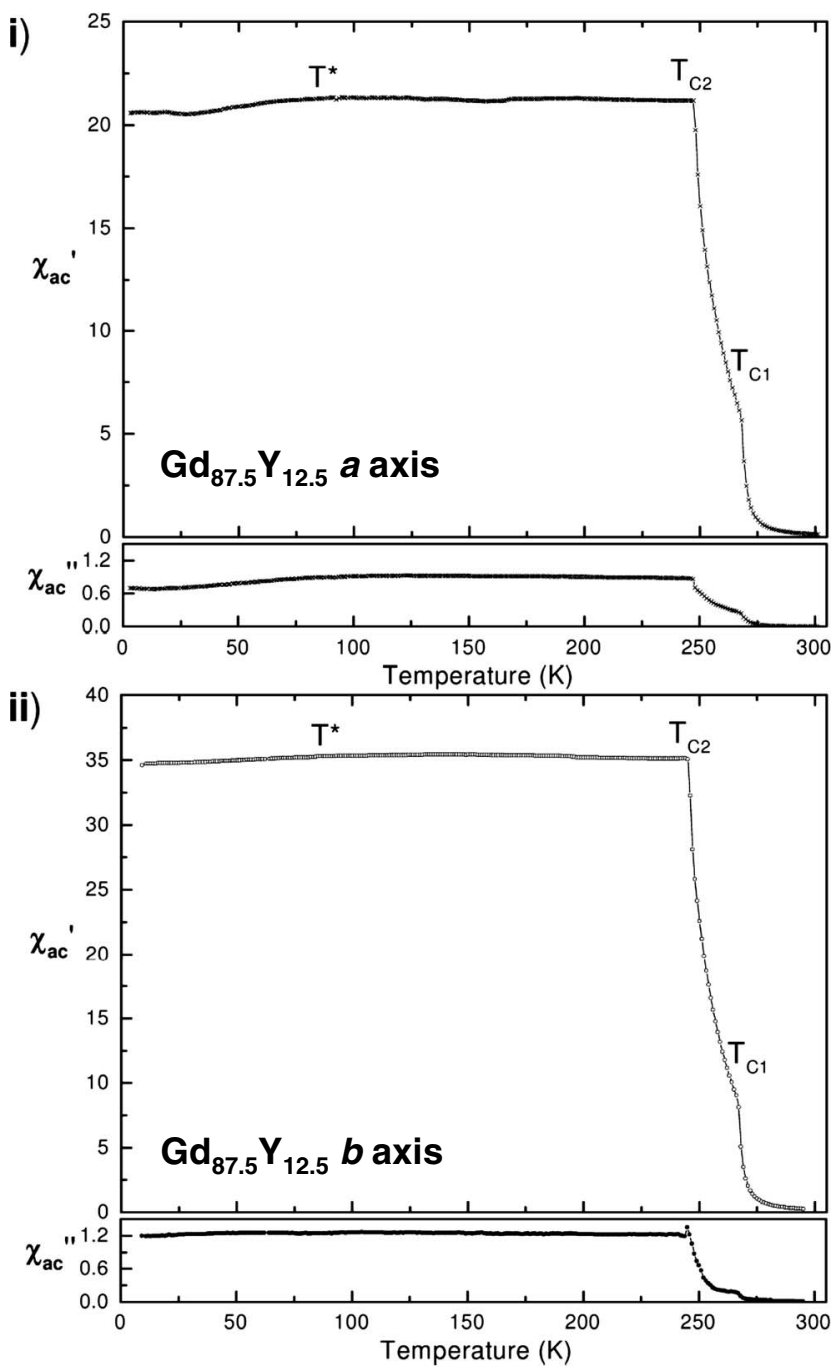

FIG. 1. The external $\chi^{\prime}$ and $\chi^{\prime \prime}$ as measured on warming for the (i) $a$ and (ii) $b$ axis needles in $\operatorname{Gd}_{87.5} \mathrm{Y}_{12.5} \cdot H_{\omega}=40 \mathrm{~A} \mathrm{~m}^{-1}$, parallel to the axis measured, and with $f=113 \mathrm{~Hz}$.

$d T / d t$ on cooling and warming affected the rate of data collection.

\section{B. Magnetization}

SQUID magnetization measurements were performed to see whether the behavior observed in the ac susceptibility is evident in the dc magnetization. The low-field measurements of the temperature dependence of the magnetization $M(T)$ gave results that are in excellent agreement with the trends observed in the susceptibility. Figures 5 and 6 show $M(T)$ measured for the Gd $a$ axis needle in low applied fields and for the $\mathrm{Gd}_{97.5} \mathrm{Y}_{2.5} a$ axis needle in a range of applied fields, respectively. From these two figures it can be seen that the concave-upward growth is evident at low fields and that for the $\mathrm{Gd}_{97.5} \mathrm{Y}_{2.5}$ needle, which has a shape demagnetizing factor of $N<0.03$ where the susceptibility diverges (Table I), the behavior is suppressed in applied fields $\geq 40 \mathrm{kA} \mathrm{m}^{-1}$.

\section{DISCUSSION}

For all the compositions studied, $\chi_{c}^{\prime}$ approaches $1 / N$ at $T_{C 1}$, while $\chi_{a}^{\prime}$ is limited at the onset of order and does not 

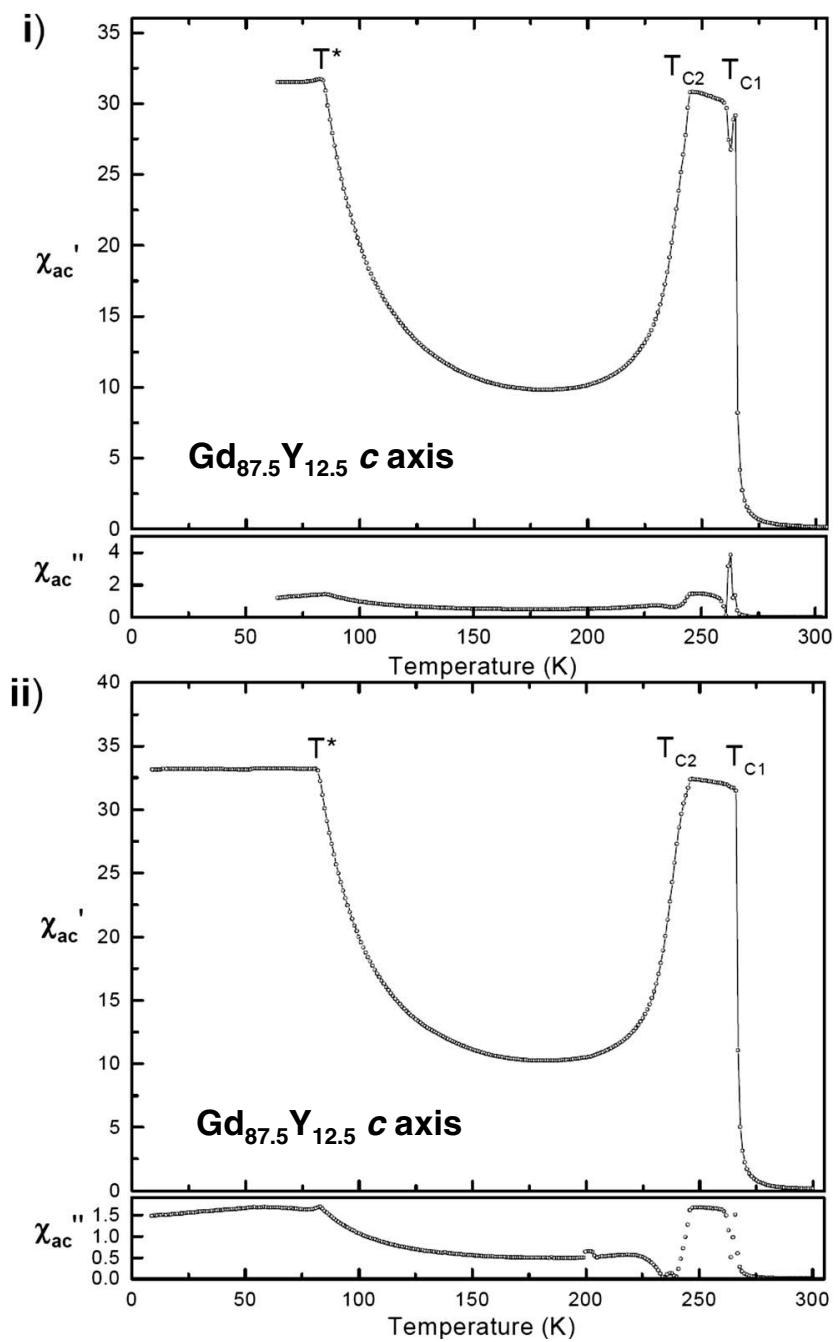

FIG. 2. The external $\chi^{\prime}$ and $\chi^{\prime \prime}$ as measured on cooling for the $c$ axis needles in $\operatorname{Gd}_{87.5} \mathrm{Y}_{12.5}$ with (i) $H_{\omega}=40 \mathrm{~A} \mathrm{~m}^{-1}$ and (ii) $H_{\omega}$ $=400 \mathrm{~A} \mathrm{~m}^{-1}$, parallel to the $c$ axis, and with $f=113 \mathrm{~Hz}$. The tenfold increase in field (where $400 \mathrm{~A} \mathrm{~m}^{-1} \simeq 5 \mathrm{Oe}$ ) is sufficient to suppress the spike in the susceptibility at $T_{C}$.

approach $1 / N$ until the spin reorientation at $T_{C 2}$. The behavior of $\chi_{a}^{\prime}$ at and below $T_{C 1}$ indicates that, compared to $\chi_{c}^{\prime}$, the basal-plane susceptibility is prevented from diverging and that the first ordered region $\left(T_{C 1}\right.$ to $\left.T_{C 2}\right)$ is predominantly $c$ axis aligned.

While it is immediately obvious that $\chi_{c}^{\prime}$ increases sharply at $T_{C 1}$, it is also clear that the theoretical maximum of $1 / N$ is never reached at $T_{C 1}$ and that $\chi_{c}^{\prime}$ continues to increase gradually on cooling below $T_{C 1}$. This is consistent with there being a basal-plane component which prevents the full divergence of the $c$ axis susceptibility, although to some extent this phenomenon can be explained by misalignments of a few degrees between the crystal axis and the applied field. ${ }^{13}$

In the region below $T_{C 2}, \chi_{c}^{\prime}$ decreases and then increases in a smooth fashion on both warming and cooling. On cooling to $T_{C 2}$ there is an increase in $\chi_{a}^{\prime}$, indicating that the $a$ axis is magnetically easier below $T_{C 2}$ than in the region above $T_{C 2}$. The same trend is exhibited by the data from the $\operatorname{Gd}_{87.5} \mathrm{Y}_{12.5} b$ axis, so it is assumed that the behavior applies to the basal plane as a whole.
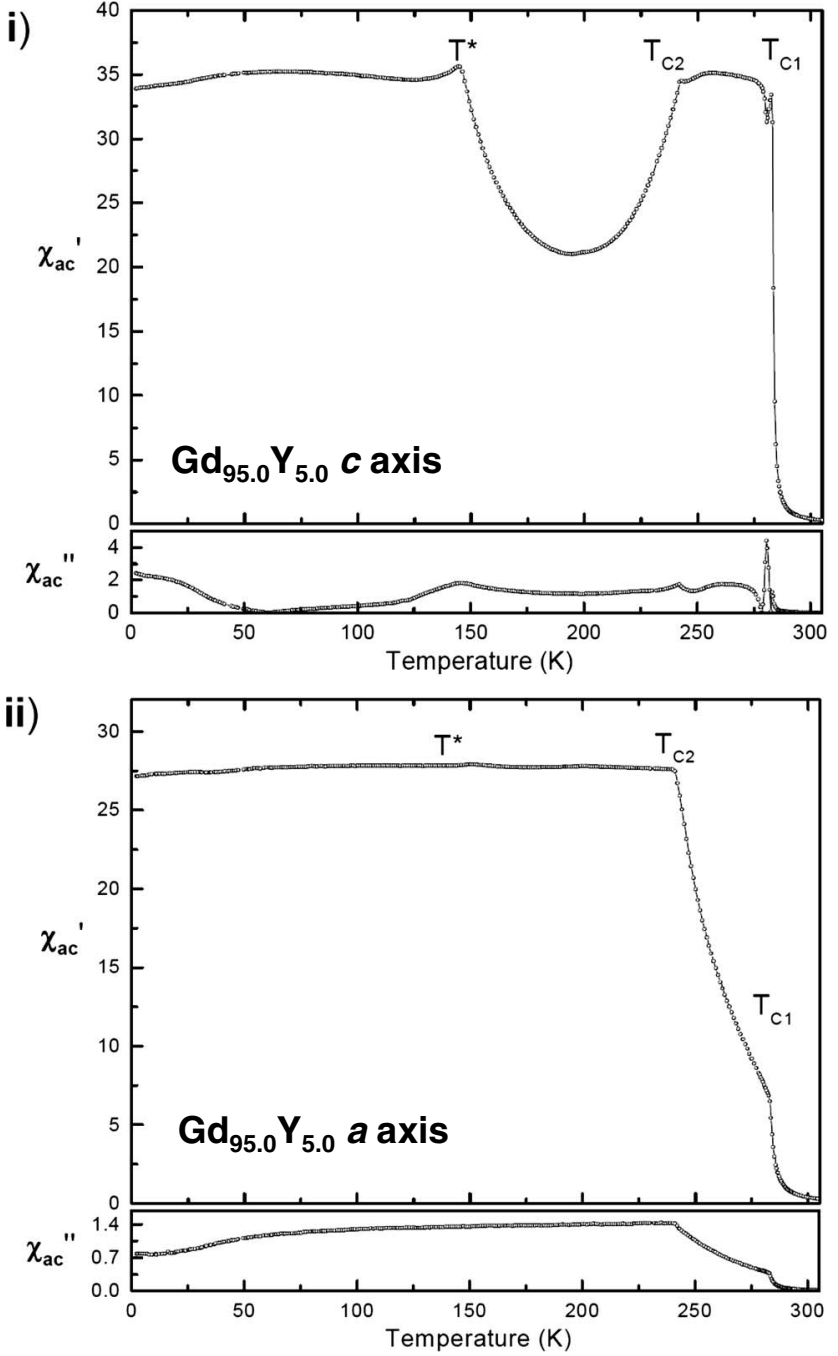

FIG. 3. The external $\chi^{\prime}$ and $\chi^{\prime \prime}$ as measured on warming for the (i) $c$ and (ii) $a$ axis needles in $\operatorname{Gd}_{95.0} \mathrm{Y}_{5.0} . H_{\omega}=40 \mathrm{~A} \mathrm{~m}^{-1}$, parallel to the axis measured, and with $f=113 \mathrm{~Hz}$.

We note that the known transitions in Gd and Gd-rich Gd-Y are usually described as occurring at $T_{C}$ and $T_{\mathrm{SR}}{ }^{12} \mathrm{We}$ have equated these transitions with $T_{C 1}$ and $T_{C 2}$ on the basis that the ordering behavior in this region appears consistent with the equivalent region for $\mathrm{Gd}_{87.5} \mathrm{Y}_{12.5}$.

In the present study, the $\mathrm{Gd}$ samples have significantly lower demagnetizing factors (as presented in Table I) than the majority of single crystals for which data have already been published. The behavior in the vicinity of $T_{C 1}$ observed for this sample of Gd is broadly consistent with the Gd-rich alloys and with the majority of the existing literature that reports $\mathrm{Gd}$ to be a $c$ axis ferromagnet in the region from $T_{C 1}$ to $T_{C 2}$.

However, while ferro I has in the past been identified by the apparent double ferromagnetism, the results from this study indicate that the characteristic behavior of the basalplane component (at a temperature significantly below $T_{C 1}$ ) is present in all the compositions studied. In $\mathrm{Gd}$, a slow buildup of magnetic order, characteristic of a disorder-order transition, is evident in the basal-plane data in the cooling approach to $T_{C 2}$, and therefore the ferro-I-like behavior may 
i )

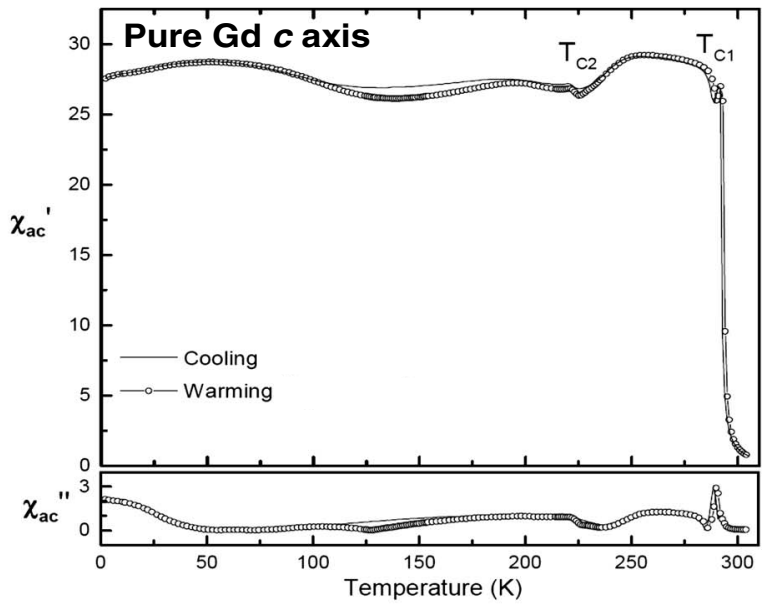

ii )

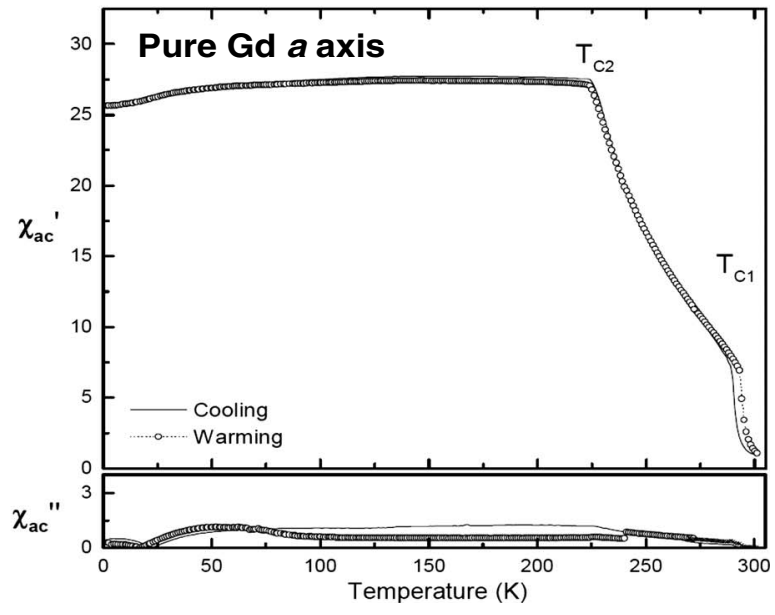

FIG. 4. The external $\chi^{\prime}$ and $\chi^{\prime \prime}$ as measured along the (i) $c$ and (ii) $a$ axis needles (warming) in pure Gd. $H_{\omega}=40 \mathrm{~A} \mathrm{~m}^{-1}$, parallel to the axis measured, and with $f=113 \mathrm{~Hz}$.

not be exclusive to the alloys. This idea is tested in Fig. 7, where $1 / \chi_{a}^{\prime}$ is plotted against temperature in the region of interest

The Curie-Weiss behavior exhibited by a ferromagnet in the region above the order-disorder transition should give rise to a constant positive gradient as a function of temperature in the inverse susceptibility in the paramagnetic region, and from Fig. 7 it is evident that $\chi_{a}^{\prime}$ exhibits Curie-Weisstype behavior in the lower-temperature region of the first ordered phase. The temperature region over which this occurs contracts from about 30 to $10 \mathrm{~K}$ with increasing Y content since the region over which the phase extends similarly contracts. There is no evidence here to suggest that, for $\mathrm{Gd}-\mathrm{Y}$ with $\mathrm{Y} \leq 10 \%, \chi_{a}^{\prime}$ exhibits anything other than the constant positive gradient, which corresponds to the concave-upward growth of the susceptibility and magnetization on cooling into the second ordered phase at $T_{C 2}$.

If the concave-upward growth in the $a$ axis magnetization is just a precursor to the basal-plane component that appears in the ferro II canted phase below $T_{C 2}$, then the interpretation of the initial magnetization measurements used to identify ferro I is called into question. ${ }^{2,4}$ Countering this suggestion are the results of the neutron-diffraction work by Bates et al. ${ }^{6}$ which support the ferro I interpretation of these early mag-

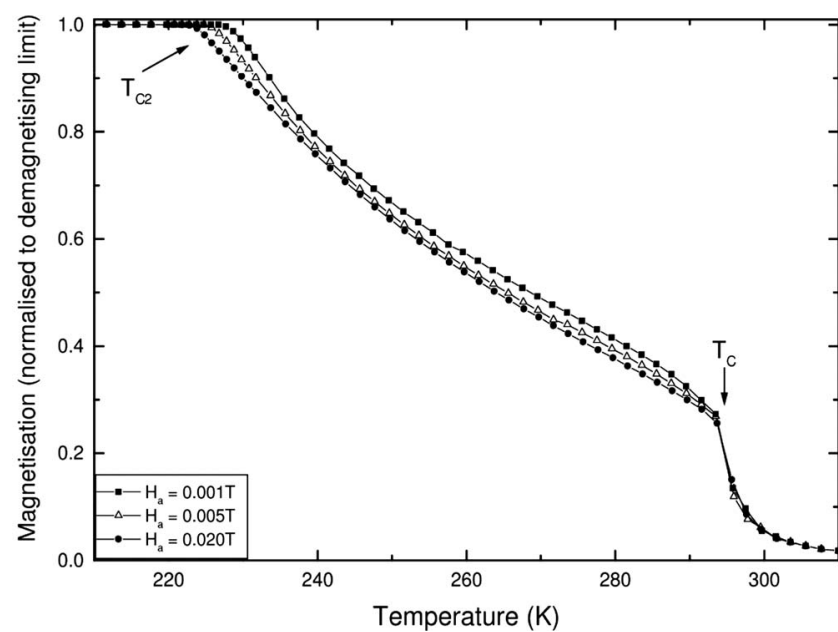

FIG. 5. The isofield $M(T)$ behavior for the pure $\mathrm{Gd} a$ axis needle, with $H_{a}$ aligned parallel to the needle axis. These data contradict the assumption that pure Gd exhibits concave-downward growth in the approach to $T_{C 2}$ and support the possibility that ferro I may be present in the element.

netization measurements. If the concave-upward growth can be interpreted as an indication of the ferro I phase, then it appears that there is a randomized basal-plane component in the first ordered region of $\mathrm{Gd}$, and the spin-reorientation may be related both to the development of the canting angle and to the ordering of the basal-plane component. This latter ordering process could in part account for unusual features noted in previous studies of $\mathrm{Gd}$, such as the small critical field required for the suppression of the spin-reorientation transition in the basal plane ${ }^{14}$ and the significant changes in the basal-plane properties at $T_{C 2}$ in pure Gd. For example, as shown in careful studies of the electrical transport properties of this alloy series, ${ }^{12}$ the $a$ axis resistivity drops on cooling through $T_{C 2}$ indicating a significant increase in magnetic order.

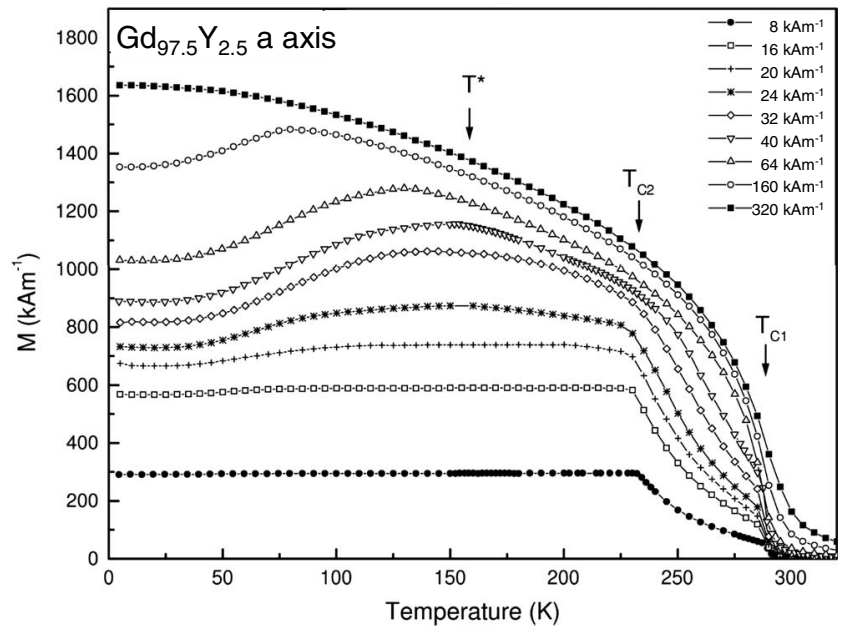

FIG. 6. The isofield $M(T)$ behavior for the $\operatorname{Gd}_{97.5} \mathrm{Y}_{2.5} a$ axis needle, with $H_{a}$ aligned parallel to the needle axis. The transition temperatures observed for measurements in zero-applied field are included for reference. The concave-upward growth in the $a$ axis moment is evident in the approach to $T_{C 2}$ for applied fields up to $40 \mathrm{kA} \mathrm{m}^{-1}$. 


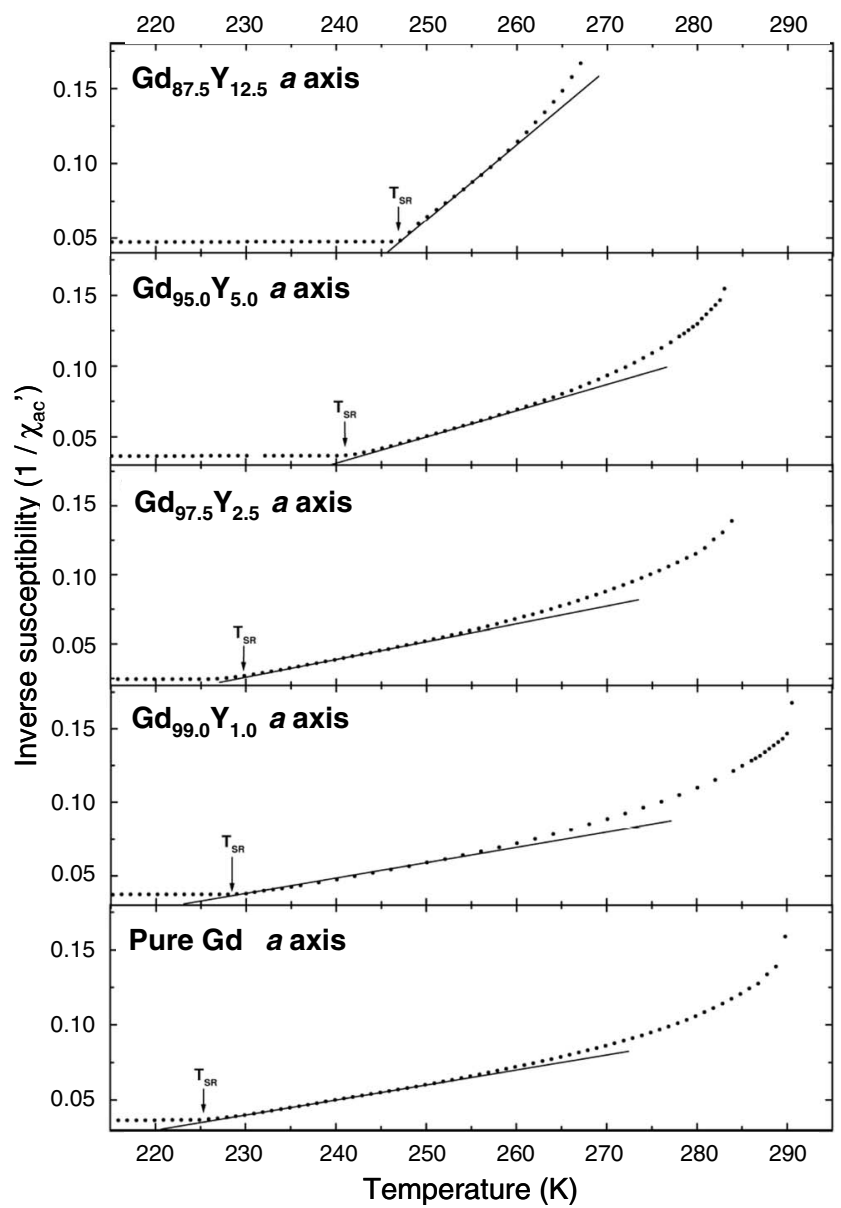

FIG. 7. The behavior of the inverse susceptibility is Curie-Weiss type for the $a$ axis susceptibility in the region between $T_{C 1}$ and $T_{C 2}$, and straight lines have been added to each plot of the inverse susceptibility to indicate the range over which the gradient is constant. The Curie-Weiss-type behavior is strong evidence to suggest that the basal-plane component goes through an order-disorder transition at $T_{C 2}$, which is a signature of ferro I.

Gd sits at the center of the rare-earth series, with a halffilled $4 f$ shell. The Gd ion is therefore in an $S$ state, with a spherical charge cloud, giving Gd comparatively low magnetocrystalline anisotropy among the rare earths. One consequence of this is that slight variations in crystal quality and purity can have a strong influence on magnetic order and transition temperature. Previous experimental observations of unusual ordering behavior in Gd have been attributed to oxide platelet inclusions, ${ }^{15-18}$ where start material purity and crystal age may have been contributing factors. The samples in the present study were all grown from high-purity start materials as detailed in Sec. II A, stored in a desiccator, and studied within a period of 24 months after crystal growth. No difference was observed in the measured magnetic properties during this period. We compared the Gd sample from this series with an earlier study of a high-purity Gd crystal grown from a different batch of start material. ${ }^{19}$ Here, a highresolution determination of the Curie temperature was made from measurement of the elastic constant $C_{33}$ using the pulse-echo-overlap technique. The data are shown in Fig. 8 where the accuracy in temperature was within $10 \mathrm{mK}$. The

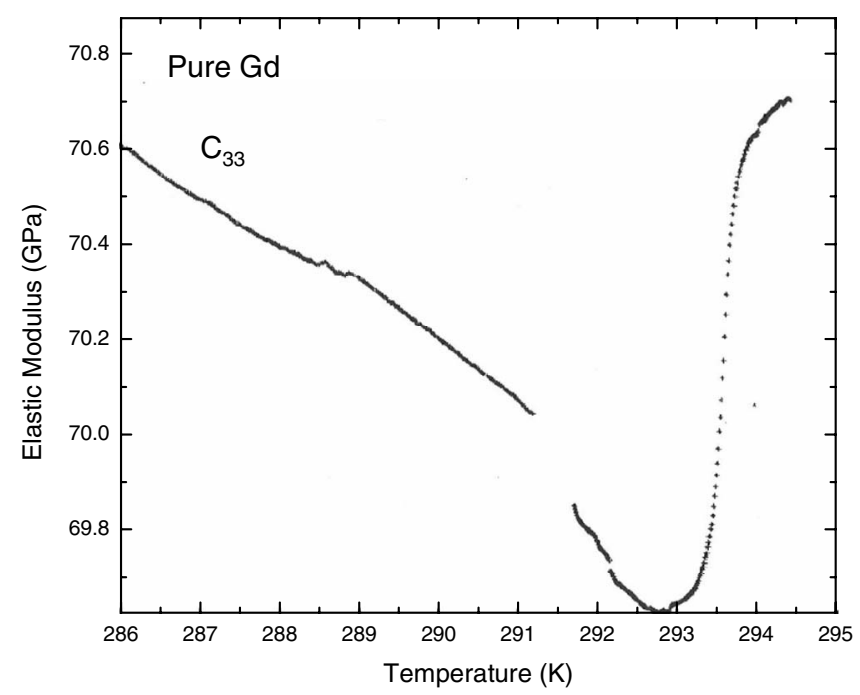

FIG. 8. Measurement of the $C_{33}$ elastic constant for a highpurity sample of $\mathrm{Gd}$, obtained using the ultrasound pulse-echooverlap technique. Temperature control was within $1 \mathrm{mK}$, with an absolute accuracy of $10 \mathrm{mK}$. The rate of change in temperature was $2 \mathrm{~K} / \mathrm{h}$. Adapted from Fig. 8.3 in Ref. 19.

value of the Curie temperature $T_{C}$, which was assumed to coincide with the minimum in $C_{33}$, occurred at $292.85 \pm 0.05 \mathrm{~K}$, where the uncertainty is due largely to identifying the location of the minimum in $C_{33}$ rather than the accuracy of the temperature measurement. Considering that the samples were prepared from independent start materials, the value from the high-resolution elastic constant measurement is in good agreement with the value of $T_{C 1}$ $=293.3 \pm 0.3 \mathrm{~K}$ obtained from the Gd crystals used in the present study. ${ }^{18}$

The apparent presence of double ferromagnetism in $\mathrm{Gd}$ undermines the ferro I models based on the concentration effects of $\mathrm{Y}$ in $\mathrm{Gd}$. If we consider the chemical concentration variation model further, it appears to break down on the microscopic scale where it relies on a reduction in the meanfree path with the introduction of Y. This is because the resistivity of $\mathrm{Y}$ is significantly lower than that of $\mathrm{Gd}$ due to the absence of spin-dependent scattering. Although alloying Gd with Y modifies the band structure and increases the residual resistivity, the total resistivity is significantly reduced. The mean-free path should therefore increase with the introduction of $\mathrm{Y}$, eventually favoring next-nearest-neighbor ordering over nearest neighbor to the extent that the basalplane helix develops. ${ }^{20}$

The presence of ferro I in Gd is not straightforward to justify given that the existing experimentally determined anisotropy constants for this phase indicate that first anisotropy constant $K_{1}$ is positive and that the higher-order terms are negligible. Despite this, the most comprehensive anisotropy data for Gd (Ref. 21) suggest an energy minimum that extends $\pm 5^{\circ}$ either side of the $c$ axis. From these data it cannot be determined whether the minimum-energy point coincides with the $c$ axis or whether the minimum is at a slight canting angle $\theta$. If an energy minimum either side of the $c$ axis also occurs in the Gd-rich Gd-Y alloys, this could in part account for the development of the ferro I phase. In the existing 
phase diagram no attempt has been made to indicate the transition from ferro I to a simple $c$ axis ferromagnet in the approach to pure Gd, but this would be unnecessary if ferro $\mathrm{I}$ is also present in $\mathrm{Gd}$.

The question of whether gadolinium is truly ferromagnetic arose in a comparatively recent ac susceptibility study. ${ }^{22}$ While our data are consistent with the main body of formerly published work on $\mathrm{Gd}$ and $\mathrm{GdY}$, our $c$ - and $a$-axis results are not entirely consistent with the experimental observations of Coey et al. ${ }^{22}$ It was not possible to confirm the precise reasons for these differences

In summary, the results presented here for Gd do not, on balance, appear to contradict the evidence in the existing literature. The suggestion that Gd typically exhibits concavedownward growth in the approach to $T_{C 2}$ (Ref. 2) is actually quite hard to justify, as the majority of the data are ambiguous due to being severely limited by high demagnetizing factors, and additionally there is evidence of concave-upward growth in the approach to $T_{C 2}$ in basal-plane samples of $\mathrm{Gd}$ in both early ${ }^{23}$ and recent studies.

\section{CONCLUSIONS}

Our measurements of low-field ac susceptibility and magnetization for high-quality Gd and Gd-Y single crystals indicate an ordered $c$ axis component and an effectively disordered basal-plane component in the first magnetically ordered phase for each composition. This suggests that either the double concave-upward growth, identified as one of the signatures of ferro I in previous studies, is not necessarily an indicator of the phase, or that ferro I is present in pure $\mathrm{Gd}$ as well as the alloys. Of the several models that exist to describe ferro I, the presence of ferro I in pure Gd would favor the random-cone model and rule out models based on chemical concentration variations.

\section{ACKNOWLEDGMENTS}

One of the authors (J.F.C.) acknowledges EPSRC for support of this work. We would like to thank D. Fort for the growth and preparation of the single crystals used in this study. *joanna.collingwood@physics.org

${ }^{1}$ S. Legvold, T. Ito, and B. J. Beaudry, Phys. Rev. Lett. 45, 1275 (1980).

${ }^{2}$ T. Ito, S. Legvold, and B. J. Beaudry, Phys. Rev. B 23, 3409 (1981).

${ }^{3}$ S. Bates, Ph.D. thesis, University of Hull, 1985.

${ }^{4}$ T. Ito, M. Oka, S. Legvold, and B. J. Beaudry, Phys. Rev. B 29, 6276 (1984).

${ }^{5}$ R. Melville, Ph.D. thesis, University of Warwick, 1989.

${ }^{6}$ S. Bates, G. McIntyre, S. Palmer, and J. Sousa, J. Phys. F: Met. Phys. 17, 1973 (1987)

${ }^{7}$ R. Eccleston, S. Brown, and S. Palmer, J. Magn. Magn. Mater. 140-144, 745 (1995).

${ }^{8}$ D. Fort, J. Alloys Compd. 177, 31 (1991).

${ }^{9}$ D. Chen, J. Brug, and R. Goldfarb, IEEE Trans. Magn. 27, 3601 (1991).

${ }^{10}$ J. Hopkinson, Philos. Trans. R. Soc. London 180, 443 (1889).

${ }^{11}$ J. Collingwood, J. Santos, J. Araujo, J. Sousa, J. Moreira, C. Lim, C. Edwards, D. Fort, and S. Palmer, IEEE Trans. Magn. 37, 2166 (2001).
${ }^{12}$ J. Collingwood, C. Edwards, S. Palmer, J. Santos, and J. Sousa, J. Appl. Phys. 93, 8349 (2003).

${ }^{13}$ S. N. Kaul and S. Srinath, Phys. Rev. B 62, 1114 (2000).

${ }^{14}$ M. Salamon and D. Simons, Phys. Rev. B 7, 229 (1973).

${ }^{15}$ R. Smith, B. Tanner, and W. Corner, J. Phys. F: Met. Phys. 7, L229 (1977).

${ }^{16}$ K. Belov and A. Ped'Ko, Sov. Phys. JETP 15, 62 (1962).

${ }^{17}$ K. Tohyama and S. Chikazumi, J. Phys. Soc. Jpn. 35, 47 (1973).

${ }^{18}$ J. Collingwood, Ph.D. thesis, University of Warwick, 2003.

${ }^{19}$ D. Jiles, Ph.D. thesis, University of Hull, 1979.

${ }^{20}$ S. Legvold, Ferromagnetic Materials (North-Holland, Amsterdam, 1980), Vol. 1.

${ }^{21}$ R. Smith, W. Corner, B. Tanner, R. Jordan, and D. Jones, in Rare Earths and Actinides, Conference Series No. 37 (The Institute of Physics, 1978).

${ }^{22}$ J. Coey, V. Skumryev, and K. Gallagher, Nature (London) 401, 35 (1999).

${ }^{23}$ H. Nigh, S. Legvold, and F. Spedding, Phys. Rev. 132, 1092 (1963) 